\title{
Nodal superconducting-gap structure in ferropnictide superconductor $\mathrm{BaFe}_{2}\left(\mathrm{As}_{0.7} \mathrm{P}_{0.3}\right)_{2}$
}

\author{
Y. Zhang, Z. R. Ye, Q. Q. Ge, F. Chen, Juan Jiang, M. Xu, B. P. Xie and D. L. Feng*
}

\begin{abstract}
The superconducting-gap distribution is a pivotal characteristic for a superconductor. Whereas the cuprates and conventional phonon-mediated superconductors are characterized by distinct $d$-wave and $s$-wave pairing symmetries with nodal and nodeless gap distributions respectively, the superconductinggap distributions in iron-based superconductors are rather diversified. Whereas nodeless gap distributions have been directly observed in $\mathrm{Ba}_{1-x} \mathrm{~K}_{x} \mathrm{Fe}_{2} \mathrm{As}_{2}, \mathrm{BaFe}_{2-x} \mathrm{Co}_{x} \mathrm{As}_{2}, \mathrm{~K}_{x} \mathrm{Fe}_{2-y} \mathrm{Se}_{2}$ and $\mathrm{FeTe}_{1-x} \mathrm{Se}_{x}$ (refs 1-4), the signatures of nodal superconducting gaps have been reported in LaOFeP, LiFeP, $\mathrm{KFe}_{2} \mathrm{As}_{2}$, $\mathrm{BaFe}_{2}\left(\mathrm{As}_{\mathbf{1}_{-x}} \mathrm{P}_{x}\right)_{2}, \mathrm{BaFe}_{2-x} \mathrm{Ru}_{x} A s_{2}$ and FeSe (refs 5-12). We here report the angle-resolved photoemission spectroscopy measurements on the superconducting-gap structure of $\mathrm{BaFe}_{2}\left(\mathrm{As}_{0.7} \mathrm{P}_{0.3}\right)_{2}$, and in particular the direct observation of a circular line node on the largest hole Fermi surface around the $Z$ point at the Brillouin zone boundary. Our findings rule out a $d$-wave-pairing origin for the nodal gap, and establish the existence of nodes in iron pnictides under the $s$-wave pairing symmetry.
\end{abstract}

The pairing symmetry of the Cooper pair in a superconductor is manifested in an energy gap of its single-particle excitation spectrum. In particular, nodes (that is zero gaps) or line nodes of the superconducting gap often imply unconventional pairing symmetries. For most iron-based superconductors, there are electron Fermi surfaces (FSs) at the Brillouin zone corner and hole FSs at the zone centre. It has been proposed that the pairing interactions between the electron and hole FSs will induce a nodeless $s$-wave order parameter with opposite signs on them ${ }^{13-15}$. Whereas this nodeless $s^{ \pm}$-wave pairing symmetry has gained increasing experimental support for some compounds ${ }^{16-18}$, an increasing number of reports on the nodal gap of others pose serious challenges $^{5-12}$ and debates ${ }^{19-25}$. So far, the key to resolving this issue, namely the location of the nodal gap, remains unknown.

We have made angle-resolved photoemission spectroscopy (ARPES) measurements on $\mathrm{BaFe}_{2}\left(\mathrm{As}_{0.7} \mathrm{P}_{0.3}\right)_{2}$ with a superconducting transition temperature $\left(T_{\mathrm{c}}\right)$ of $30 \mathrm{~K}$ (Methods). As previous detailed polarization-dependent study has shown ${ }^{26}$ and as reproduced in Fig. 1a, there are three hole FSs $(\alpha, \beta$ and $\gamma)$ surrounding the central $\Gamma-\mathrm{Z}$ axis of the Brillouin zone, and two electron FSs $(\delta$ and $\eta$ ) around the corner. Near $\Gamma$, the $\alpha$ and $\beta$ FSs are coincident, and they are mainly composed of Fe $d_{x z}$ and $d_{y z}$ orbitals. The $\gamma$ band is composed of the $d_{x y}$ orbital, and shows little $k_{z}$ dispersion ${ }^{26}$. The $\alpha$ FS exhibits a large warping along $k_{z}$, and becomes the outmost FS around $Z$ (refs 26,27). To examine the superconducting gap, Fig. $1 \mathrm{~b}$ shows the symmetrized photoemission intensity in the normal and superconducting state taken along cut 1 across $\Gamma$. The temperature-broadening effect of the Fermi-Dirac distribution on the spectrum is minimized by symmetrizing. The spectral weight near the Fermi energy $\left(E_{\mathrm{F}}\right)$ is suppressed in the superconducting state. More specifically, the temperature dependences of the symmetrized photoemission spectra at the Fermi crossings of the $\alpha / \beta$ and $\gamma$ bands are shown in Fig. 1c. Above $T_{c}$ the spectral lineshape does not change noticeably, but below $T_{\mathrm{c}}$ the spectral weight near $E_{\mathrm{F}}$ is suppressed quickly because of the opening of a superconducting gap with decreasing temperature. A small coherent peak can be observed at $8 \mathrm{~K}$. By fitting the spectra with a typical superconducting-state spectral function (Fig. 1c), we could obtain the temperature dependence of the superconducting gap in Fig. 1d, which can be fitted well to the Bardeen-Cooper-Schrieffer formula (solid line). Similarly, the opening of the gap on the $\delta$ and $\eta$ FSs is illustrated in Fig. 1e-g for $k_{z}=0.54 \pi$. The extrapolated zero-temperature gaps give $2 \Delta_{0} / k_{\mathrm{B}} T_{\mathrm{c}} \approx 5-6$ for $\mathrm{BaFe}_{2}\left(\mathrm{As}_{0.7} \mathrm{P}_{0.3}\right)_{2}$.

Figure 2 presents an extensive survey on the possible gap nodes on electron FSs following the proposal of a recent angle-dependent transport study ${ }^{7}$. Figure 2 a shows four typical cuts in the $k_{z}=0.5 \pi$ plane. The isotropic superconducting gaps open on all the Fermi crossings. Similar data are measured for different $k_{z}$ values. The superconducting-state spectra are shown in Fig. $2 \mathrm{~b}$ along three cross-sections of the $\eta$ FS in $k_{z}=0,0.5 \pi$ and $\pi$ planes, and in Fig. 2c for its $k_{z}-k_{y}$ cross-section. The leading-edge gap opening can be clearly observed with little $k_{z}$ dependence from the raw spectra in Fig. 2d. Similarly, Fig. 2d-f plot the spectra on the $\delta$ FS. In addition, the data taken in the $k_{z}=0.2 \pi, 0.34 \pi, 0.54 \pi$, $0.64 \pi$ and $0.8 \pi$ planes are shown in Supplementary Fig. S5. The superconducting gaps are always finite in all cases. If the gap nodes were continuously located along certain vertical lines or loops on the FS as suggested ${ }^{7,19-21}$, they should not be missed in such an extensive search. We would have observed at least some variation of the gap if not nodes, owing to the finite momentum resolution. The fact that the superconducting gap is finite and almost fixed to the same size over the entire $\delta$ (or $\eta$ ) FS proves that the line nodes of the gap are absent from the electron FSs.

It was predicted that sufficient phosphorus doping in $\mathrm{BaFe}_{2}\left(\mathrm{As}_{1-x} \mathrm{P}_{x}\right)_{2}$ would shift the $d_{x y}$-based $\gamma$ band below $E_{\mathrm{F}}$, and consequently vertical line nodes would appear in the electron FS (refs 19-21). The fact that $\gamma$ does not sink below $E_{\mathrm{F}}$, and the absence of vertical nodes on the electron FSs, all indicate that this is not the case.

Figure 3 gives the detailed map of superconducting gaps on the hole FSs. The symmetrized spectra for different $k_{z}$ values along the $\beta, \gamma$ and $\alpha$ FSs are shown in Fig. 3a-c respectively. As indicated by the dashed line, the gap decreases slightly from $k_{z}=0$ to $k_{z}=\pi$ on $\beta$ and $\gamma$, but much more remarkably on $\alpha$, and it even closes near the $\mathrm{Z}$ point. The nodal superconducting 

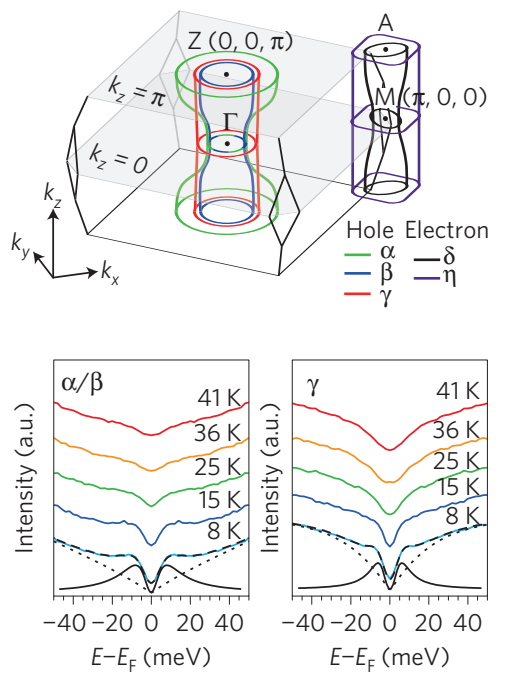

b

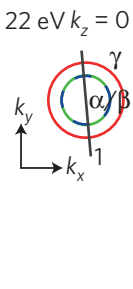

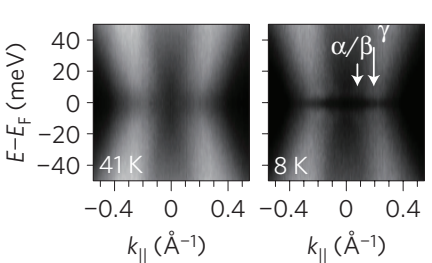

Low High

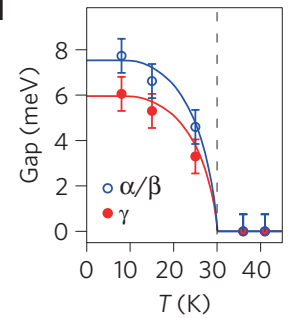

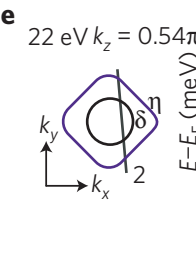

f
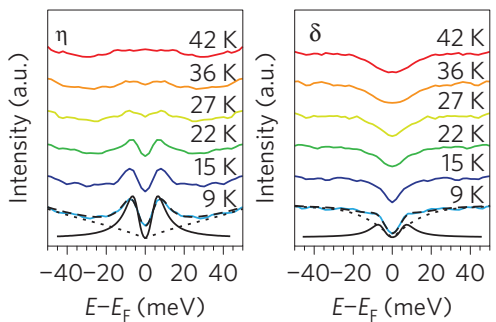

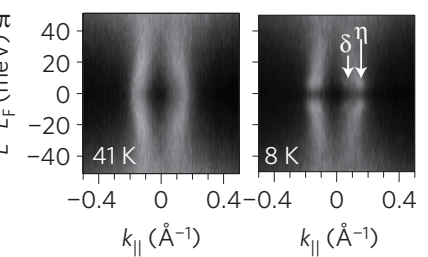

Low High

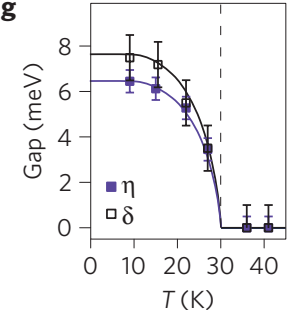

Figure 1 | The temperature dependence of the photoemission data of $\mathbf{B a F e}_{\mathbf{2}}\left(\mathrm{As}_{0.7} \mathbf{P}_{\mathbf{0 . 3}}\right)_{2}$. $\mathbf{a}$, The three-dimensional FSs of $\mathrm{BaFe} 2\left(\mathrm{As} 0.7 \mathrm{P}_{0.3}\right)_{2}$. The two-iron unit cell is implemented here, with the Fe-Fe direction as the $k_{x}$ direction. The electron FSs are only illustrated at one corner of the Brillouin zone for simplicity. $\mathbf{b}$, The photoemission intensity taken around the zone centre with $22 \mathrm{eV}$ photons at 41 and $8 \mathrm{~K}$. $\mathbf{c}$, The temperature dependence of the symmetrized spectra measured at the Fermi crossings of $\alpha / \beta$ and $\gamma$ along the cut in $\mathbf{b}$. Two examples of the fitted curve, background and superconducting

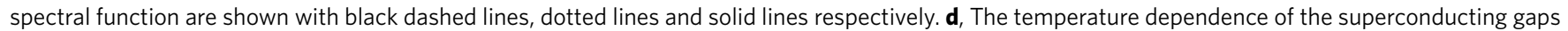

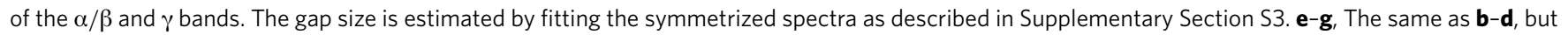
taken around the zone corner along cut 2 with $22 \mathrm{eV}$ photons. The error bars in $\mathbf{d}$ and $\mathbf{g}$ are standard deviations of the measured superconducting gaps.
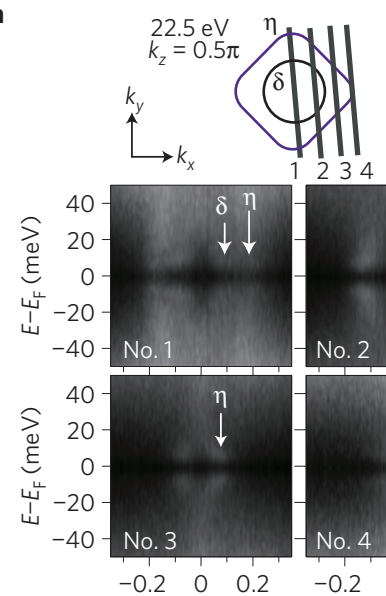
$k_{\|}\left(\AA^{-1}\right)$

d
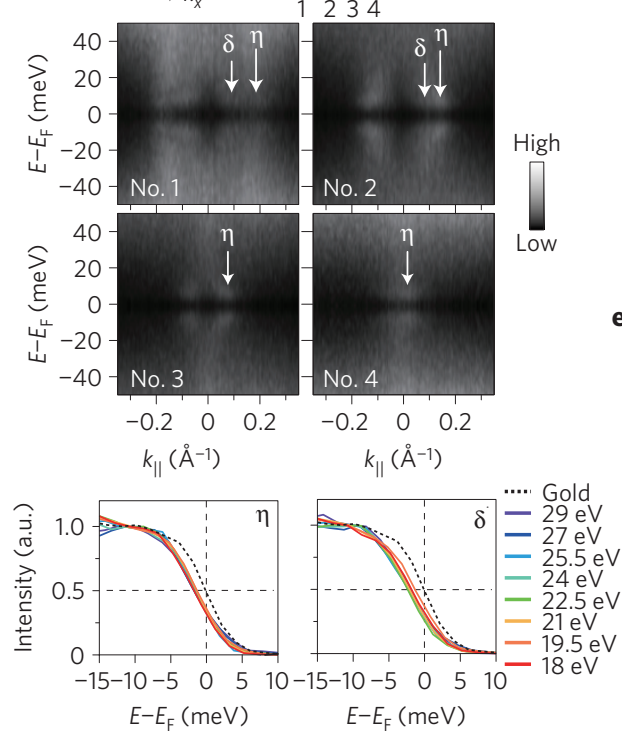

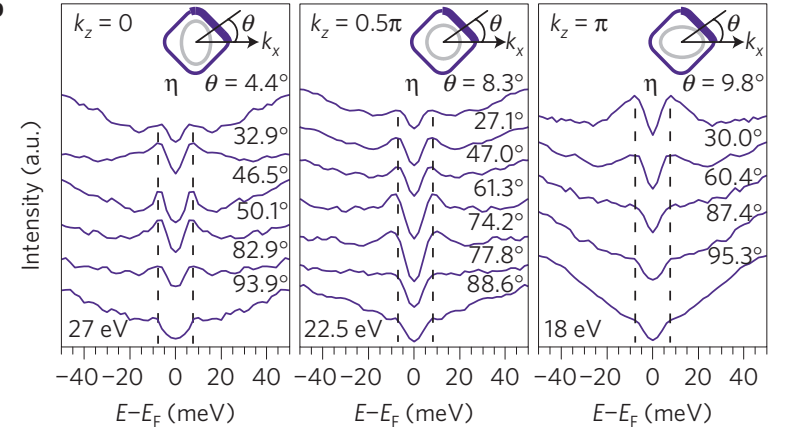

e

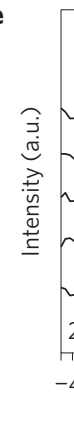$$
E-E_{\mathrm{F}}(\mathrm{meV})
$$$$
E-E_{\mathrm{F}}(\mathrm{meV})
$$

$E-E_{\mathrm{F}}(\mathrm{meV})$

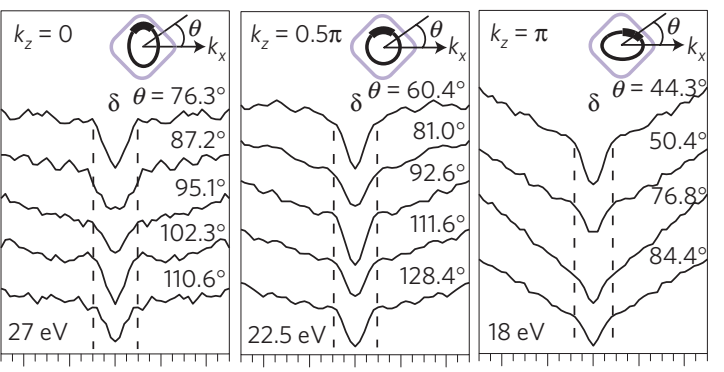

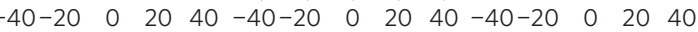
$E-E_{\mathrm{F}}(\mathrm{meV})$

$E-E_{\mathrm{F}}(\mathrm{meV})$
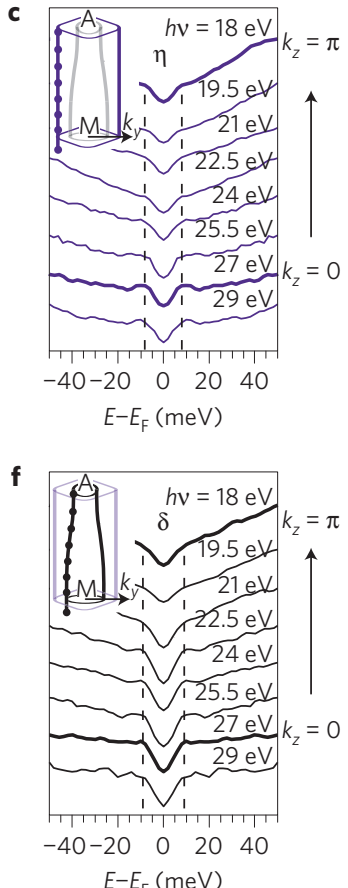

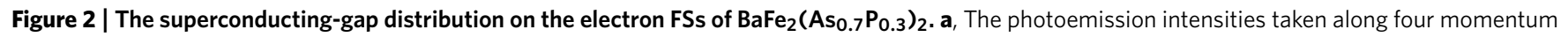
cuts near the zone corner with $22.5 \mathrm{eV}$ photons. b. The symmetrized spectra at the marked polar angles on the $\eta$ electron FS measured at three typical

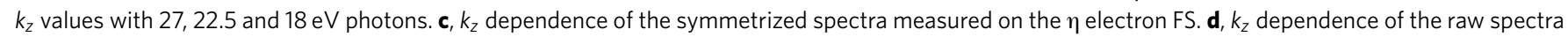
measured on the $\delta$ and $\eta$ electron FSs. The black dotted curves are reference spectra from a piece of polycrystalline gold in electrical contact with the sample. e,f, The same as $\mathbf{b}, \mathbf{c}$, but taken on the $\delta$ electron FS. The dashed curves in $\mathbf{b}, \mathbf{c}$, e and $\mathbf{f}$ are guides to the eyes for the variation of the

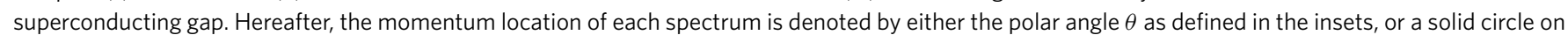
the FS. All data were taken in the superconducting state at $9 \mathrm{~K}$.

gap on the $\alpha$ FS around $\mathrm{Z}$ is further confirmed by the vanishing leading-edge gap in the corresponding spectra presented in Fig. 3d and Supplementary Fig. S4. On the other hand, the middle points of the spectral leading edges are always below $E_{\mathrm{F}}$ for the $\beta$ and $\gamma$ bands, indicating their nodeless nature. The photoemission intensity along three cuts (Fig. 3e) and the symmetrized spectra around three FS cross-sections (Fig. 3f) in the $k_{z}=\pi$ plane show that finite and isotropic gaps open on the $\beta$ and $\gamma$ FSs, and a circular line node 
a
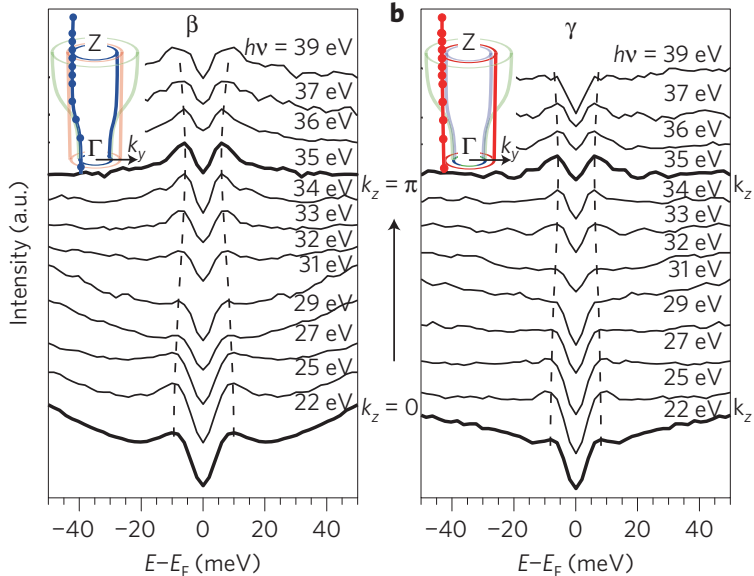

$E-E_{F}(m e V)$
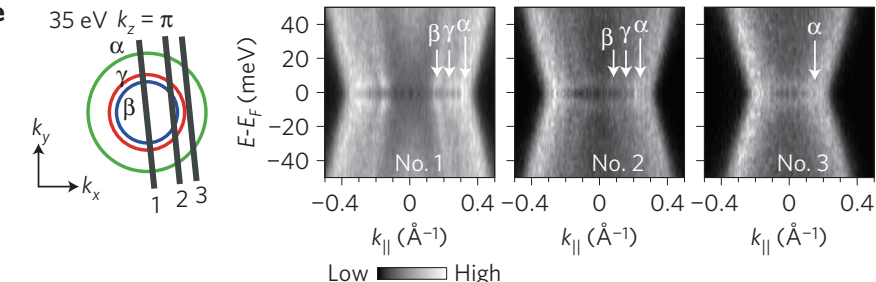

f
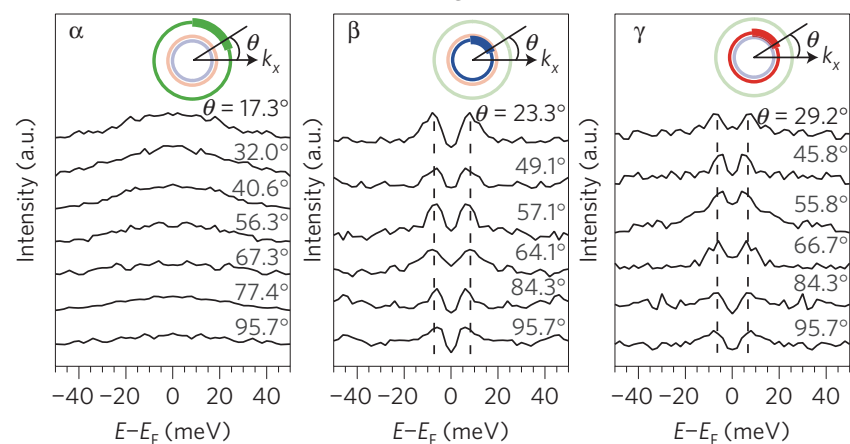
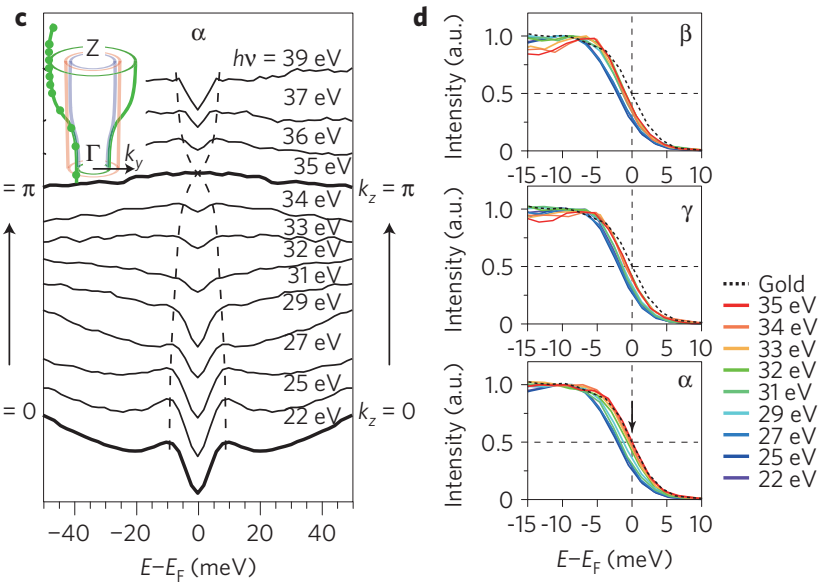

g $27 \mathrm{eV} \mathrm{k}=0.38 \pi$

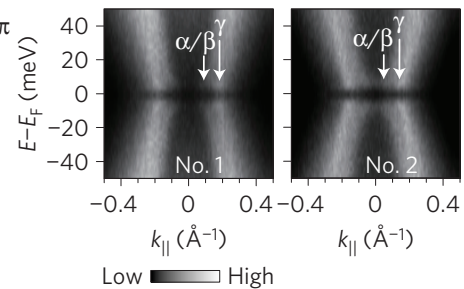

h
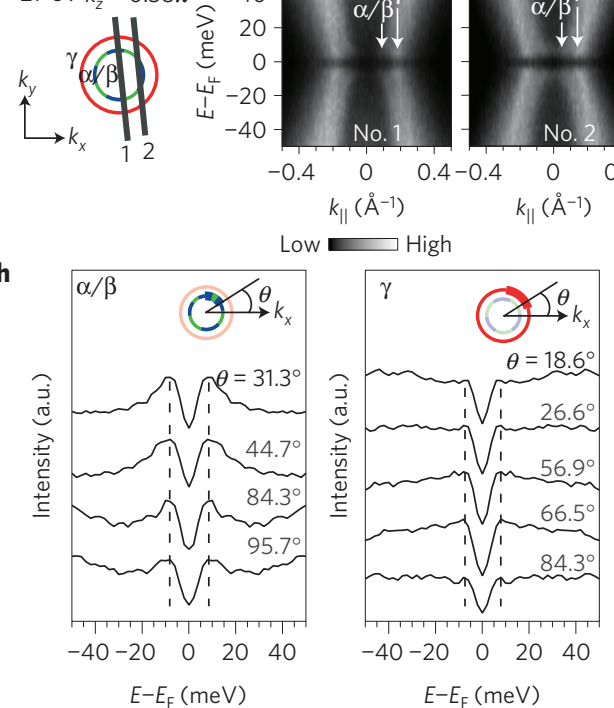

Figure 3 | The superconducting-gap distribution on the hole FSs of $\mathrm{BaFe}_{2}\left(\mathrm{As}_{0.7} \mathbf{P}_{0.3}\right)_{2} \cdot \mathbf{a}-\mathbf{c}, k_{z}$ dependence of the symmetrized spectra measured on the $\beta, \gamma$ and $\alpha$ hole FSs respectively. The symmetrized spectra near $k_{z}=0$ and $k_{z}=\pi$ are shown in thicker curves. The dashed line is a guide to the eyes for the variation of the superconducting gap at different $k_{z}$ values. $\mathbf{d}, k_{z}$ dependence of the raw spectra measured on the $\beta$, $\gamma$, and $\alpha$ hole FSs. The black dotted curves are reference spectra from a piece of polycrystalline gold in electrical contact with the sample. The leading-edge middle point only crosses $E_{\mathrm{F}}$ near $Z$ for the $\alpha$ band, as illustrated by the black arrow. Note that, as the leading-edge middle-point position underestimates the gap, the gap magnitudes were determined through the fitting procedure described in Supplementary Section S3. e, The photoemission intensities taken along three momentum cuts, and $\mathbf{f}$, the symmetrized spectra at the marked polar angles along the $\alpha, \beta$ and $\gamma$ hole FSs taken with $35 \mathrm{eV}$ photons in the $k_{z}=\pi$ plane around Z. g,h, Similar to e,f, but taken with $27 \mathrm{eV}$ photons in the $k_{z}=0.38 \pi$ plane. All data were taken in the superconducting state at $9 \mathrm{~K}$. We note that we can usually distinguish an energy gap if it is bigger than $20 \%$ of the resolution in a photoemission experiment, and the energy resolution here with $35 \mathrm{eV}$ photons is $6 \mathrm{meV}$.

exists around $\mathrm{Z}$ on the $\alpha \mathrm{FS}$. As a comparison, the data taken in the $k_{z}=0$ (Supplementary Fig. S6) and $k_{z}=0.38 \pi$ planes show a full gap for all three FSs (Fig. 3g,h). Such a horizontal line-node distribution immediately rules out the $d$-wave pairing symmetry, which would give four vertical line nodes in the diagonal directions $\left(\theta= \pm 45^{\circ}, \pm 135^{\circ}\right)$, as in the cuprates. Of note, a recent ARPES study with a $7 \mathrm{eV}$ laser on $\mathrm{BaFe}_{2}\left(\mathrm{As}_{0.65} \mathrm{P}_{0.35}\right)_{2}$ has claimed the absence of a nodal gap around $\mathrm{Z}$ (ref. 28). However, on the basis of our extensive photon-energy-dependence study, a $7 \mathrm{eV}$ photon actually measures the region with $k_{z}=0.34 \pi$, far from $\mathrm{Z}$.

The gap distribution of $\mathrm{BaFe}_{2}\left(\mathrm{As}_{0.7} \mathrm{P}_{0.3}\right)_{2}$ is summarized in Fig. $4 \mathrm{a}$, together with that of $\mathrm{Ba}_{0.6} \mathrm{~K}_{0.4} \mathrm{Fe}_{2} \mathrm{As}_{2}$ replicated from ref. 29 for comparison. The limited nodal region of $\mathrm{BaFe}_{2}\left(\mathrm{As}_{0.7} \mathrm{P}_{0.3}\right)_{2}$ explains its relatively high $T_{\mathrm{c}}$. The gaps on the $\alpha, \beta, \gamma$ and $\delta$ FSs vary noticeably with $k_{z}$ in $\mathrm{BaFe}_{2}\left(\mathrm{As}_{0.7} \mathrm{P}_{0.3}\right)_{2}$, whereas only the gap on the $\alpha$ FS exhibits significant $k_{z}$ dependence in $\mathrm{Ba}_{0.6} \mathrm{~K}_{0.4} \mathrm{Fe}_{2} \mathrm{As}_{2}$. Figure $4 \mathrm{~b}$ plots the gap of $\mathrm{BaFe}_{2}\left(\mathrm{As}_{0.7} \mathrm{P}_{0.3}\right)_{2}$ as a function of $\left|\cos \left(k_{x} a\right) \cos \left(k_{y} a\right)\right|$. There are obvious deviations from the predicted gap function of $\Delta_{0}\left|\cos \left(k_{x} a\right) \cos \left(k_{y} a\right)\right|$ of the $s^{ \pm}$-wave pairing symmetry based on a two-dimensional model ${ }^{13-16}$. Furthermore, the superconducting gaps on all FSs of $\mathrm{BaFe}_{2}\left(\mathrm{As}_{0.7} \mathrm{P}_{0.3}\right)_{2}$ and the $\alpha \mathrm{FS}$ of $\mathrm{Ba}_{0.6} \mathrm{~K}_{0.4} \mathrm{Fe}_{2} \mathrm{As}_{2}$ are plotted in Fig. $4 \mathrm{c}-\mathrm{e}$ as functions of $k_{z}$, and fitted to a phenomenological function: $\Delta_{1}\left|\cos \left(k_{x} a\right) \cos \left(k_{y} a\right)\right|+\Delta_{2} \cos \left(k_{z} c\right)$ (ref. 22). The extra term here is the $k_{z}$ suppression of the superconducting gap, which could refer to the interlayer coupling in an anisotropic layered superconductor or an orbital-dependent pairing mechanism ${ }^{22,24,25,29}$. With different $\Delta_{1}$ and $\Delta_{2}$ for each band, the gaps of all the nodeless bands, including the $\alpha$ band of $\mathrm{Ba}_{0.6} \mathrm{~K}_{0.4} \mathrm{Fe}_{2} \mathrm{As}_{2}$, could be fitted. However, the nodes and gap suppression on the $\alpha \mathrm{FS}$ in $\mathrm{BaFe}_{2}\left(\mathrm{As}_{0.7} \mathrm{P}_{0.3}\right)_{2}$ cannot be fitted well by such a formula, probably owing to the strong mixing of another orbital as discussed below.

There have been a few theoretical predictions on possible nodes near $\mathrm{Z}$ in the hole FS with strong $d_{3 z^{2}-r^{2}}$ orbital characters in iron pnictides $^{22-25}$. In particular, our findings qualitatively agree with 

$\mathrm{BaFe}_{2}\left(\mathrm{As}_{0.7} \mathrm{P}_{0.3}\right)_{2}$
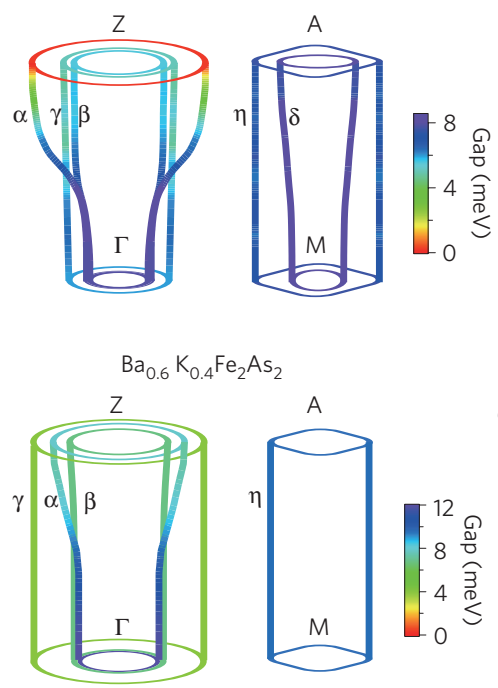

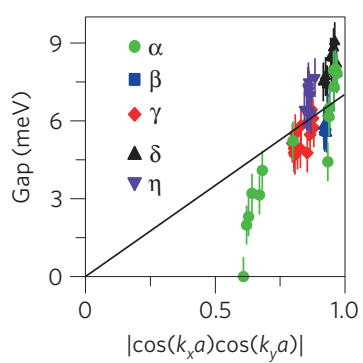

c

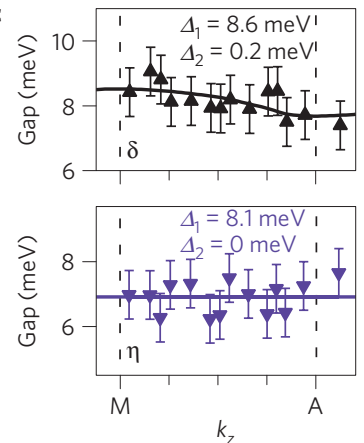

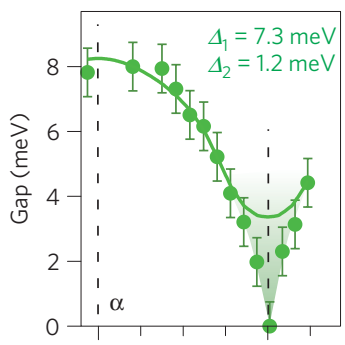
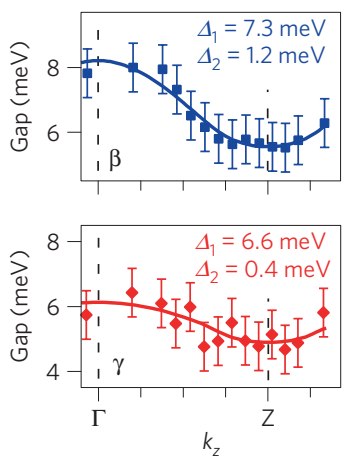

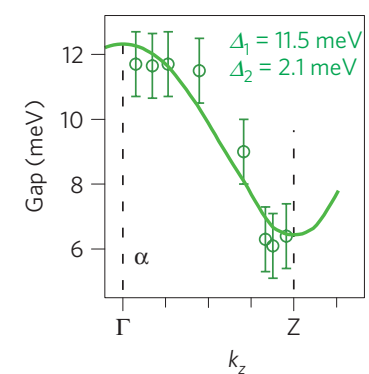

$\mathbf{f}$

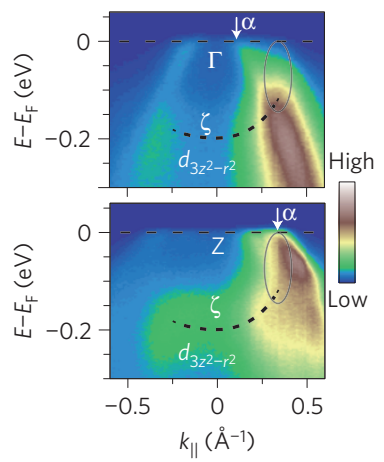

Figure 4 | Momentum dependence of the superconducting gap. a, False-colour plots of the gap distribution on the $\mathrm{FSs}_{\mathrm{S}}$ of $\mathrm{BaFe}{ }_{2}\left(\mathrm{As}_{0.7} \mathrm{P}_{0.3}\right)_{2}$ and $\mathrm{Ba}_{0.6} \mathrm{~K}_{0.4} \mathrm{Fe}_{2} \mathrm{As}_{2}$. $\mathbf{b}$ The superconducting gap of $\mathrm{BaFe}_{2}\left(\mathrm{As}_{0.7} \mathrm{P}_{0.3}\right)_{2}$ versus $\left|\cos \left(k_{x} a\right) \cos \left(k_{y} a\right)\right|$. c,d, The superconducting gaps on the $\delta$ and $\eta$ electron FSs $(\mathbf{c})$ and on the $\alpha, \beta$ and $\gamma$ hole FSs $(\mathbf{d})$ for $\mathrm{BaFe}_{2}\left(\mathrm{As}_{0.7} \mathrm{P}_{0.3}\right)_{2}$ as functions of $k_{z}$. The solid lines are fits to $\Delta_{1}\left|\cos \left(k_{x} a\right) \cos \left(k_{y} a\right)\right|+\Delta_{2} \cos \left(k_{z} c\right)$. e, The superconducting gap on the $\alpha \mathrm{FS}$ of $\mathrm{Ba}_{0.6} \mathrm{~K}_{0.4} \mathrm{Fe}_{2} \mathrm{As}_{2}$ as a function of $k_{z}$. The error bars in $\mathbf{b}$-e are standard deviations of the measured superconducting gaps. f, The band structure of $\mathrm{BaFe}_{2}\left(\mathrm{As}_{0.7} \mathrm{P}_{0.3}\right)_{2}$ near $\Gamma$ and $Z$. The ellipses indicate the spectral weight of the $\zeta$ band.

the calculations of ref. 24 on $\mathrm{BaFe}_{2}\left(\mathrm{As}_{1-x} \mathrm{P}_{x}\right)_{2}$. Those authors found that, with increasing phosphorus concentration, the $d_{3 z^{2}-r^{2}}$ orbital would be strongly mixed into the $\alpha$ band near $Z$. As a result, the superconducting gap could reverse sign on the $\alpha$ FS in the vicinity of $Z$, and thus a nodal circle emerges at the location where the sign is switched. Indeed, as pointed out in a previous study ${ }^{26}$, and also illustrated in Fig. $4 \mathrm{f}$, we do find that the $\zeta$ band with strong $d_{3 z^{2}-r^{2}}$ orbital character comes across the $\alpha$ band, and the Fermi crossing of $\alpha$ is much closer to the top of the $\xi$ band near $Z$ than near $\Gamma$, which would mix a significant amount of the $d_{3 z^{2}-r^{2}}$ orbital into $\alpha$ near $E_{\mathrm{F}}$ (more details and polarization-dependent ARPES evidence for this are presented in Supplementary Section S6). This makes sense of the intriguing observation that nodes appear in iron pnictides when the distance between the pnictogen and Fe plane $\left(h_{P n}\right)$ is less than $1.33 \AA$ (ref. 9). As for $\mathrm{BaFe}_{2}\left(\mathrm{As}_{1-x} \mathrm{P}_{x}\right)_{2}, h_{P n}$ is reduced by phosphorus doping, and it would cause larger $k_{z}$ dispersion and strong mixing of the $d_{3 z^{2}-r^{2}}$ orbital into $\alpha$.

The horizontal ring node around $\mathrm{Z}$ in $\mathrm{BaFe}_{2}\left(\mathrm{As}_{0.7} \mathrm{P}_{0.3}\right)_{2}$ is not forced by symmetry, as it is fully symmetric with respect to the point group. Therefore, it is an 'accidental' one, which is probably induced by the strong three-dimensional nature of the $\alpha$ band, for example its sizable $d_{3 z^{2}-r^{2}}$ orbital character near Z. As the superconducting order parameters at $k_{z}=0$ and $k_{z}=2 \pi$ should have the same sign, the sign of the gap function would reverse twice near $\mathrm{Z}$ instead of just once at $k_{z}=\pi$. Consequently, there should be two circular nodes located symmetrically with respect to Z (refs 24,25). With limited momentum resolution along $k_{z}$, we could not resolve such details of the nodal gap structure at present. However, it is possible that, with increasing $k_{z}$ dependence at higher phosphorus doping, these two ring nodes could be further separated, and thus resolved.

\section{Methods}

High-quality single crystals with nominal composition $\mathrm{BaFe}_{2}\left(\mathrm{As}_{0.7} \mathrm{P}_{0.3}\right)_{2}$ were synthesized through flux-free crucible growth. Shiny platelet crystals as large as $2 \times 2 \times 0.05 \mathrm{~mm}^{3}$ were obtained, which exhibit a $T_{c}$ of $30 \mathrm{~K}$ and a residual resistivity ratio of about 10 (see Supplementary Section S1 for details). Data were taken at beamline 5-4 of Stanford Synchrotron Radiation Lightsource (SSRL). All the data were taken with a Scienta electron analyser; the overall energy resolution was $5-8 \mathrm{meV}$ depending on the photon energy and the angular resolution was $0.3^{\circ}$. The samples were cleaved in situ, and measured under ultrahigh vacuum of $5 \times 10^{-11}$ torr. The out-of-plane momentum $k_{z}$ is calculated and folded into the upper half of the first Brillouin zone as described in Supplementary Section S2. It is denoted in the unit of $1 / c^{\prime}$, where $c^{\prime}$ is the distance between two neighbouring FeAs layers, which is half of the out-of-plane lattice constant $c$.

Received 9 September 2011; accepted 27 January 2012; published online 4 March 2012

\section{References}

1. Ding, H. et al. Observation of Fermi-surface-dependent nodeless superconducting gaps in $\mathrm{Ba}_{0.6} \mathrm{~K}_{0.4} \mathrm{Fe}_{2} \mathrm{As}_{2}$. Europhys. Lett. 83, 47001 (2008).

2. Terashima, K. et al. Fermi surface nesting induced strong pairing in iron-based superconductors. Proc. Natl Acad. Sci. USA 106, 7330-7333 (2009).

3. Zhang, $\mathrm{Y}$. et al. Nodeless superconducting gap in $\mathrm{A}_{x} \mathrm{Fe}_{2} \mathrm{Se}_{2}(\mathrm{~A}=\mathrm{K}, \mathrm{Cs})$ revealed by angle-resolved photoemission spectroscopy. Nature Mater. 10, 273-277 (2011).

4. Miao, H. et al. Isotropic superconducting gaps with enhanced pairing on electron Fermi surfaces in $\mathrm{FeTe}_{0.55} \mathrm{Se}_{0.45}$. Preprint at http://arxiv.org/abs/1107.0985 (2011).

5. Fletcher, J. D. et al. Evidence for a nodal-line superconducting state in LaFePO Phys. Rev. Lett. 102, 147001 (2009).

6. Hashimoto, K. et al. Line nodes in the energy gap of superconducting $\mathrm{BaFe}_{2}\left(\mathrm{As}_{1-x} \mathrm{P}_{x}\right)_{2}$ single crystals as seen via penetration depth and thermal conductivity. Phys. Rev. B 81, 220501 (2010).

7. Yamashita, M. et al. Nodal gap structure of superconducting $\mathrm{BaFe}_{2}\left(\mathrm{As}_{1-x} \mathrm{P}_{x}\right)_{2}$ from angle-resolved thermal conductivity in a magnetic field. Phys. Rev. B 84, 060507 (2011).

8. Nakai, Y. et al. ${ }^{31} \mathrm{P}$ and ${ }^{75} \mathrm{As}$ NMR evidence for a residual density of states at zero energy in superconducting $\mathrm{BaFe}_{2}\left(\mathrm{As}_{0.67} \mathrm{P}_{0.33}\right)_{2}$. Phys. Rev. B 81, 020503 (2010).

9. Hashimoto, K. et al. Nodeless vs nodal order parameters in LiFeAs and LiFeP superconductors. Preprint at http://arxiv.org/abs/1107.4505 (2011).

10. Dong, J. K. et al. Quantum criticality and nodal superconductivity in the FeAs-based superconductor $\mathrm{KFe}_{2} \mathrm{As}_{2}$. Phys. Rev. Lett. 104, 087005 (2010). 
11. Qiu, X. et al. Nodal superconductivity in $\mathrm{Ba}\left(\mathrm{Fe}_{1-x} \mathrm{Ru}_{x}\right)_{2} \mathrm{As} s_{2}$ induced by isovalent Ru substitution. Preprint at http://arxiv.org/abs/1106.5417 (2011).

12. Song, C. L. et al. Direct observation of nodes and twofold symmetry in FeSe superconductor. Science 332, 1410-1413 (2010).

13. Mazin, I. I., Singh, D. J., Johannes, M. D. \& Du, M. H. Unconventional superconductivity with a sign reversal in the order parameter of $\mathrm{LaFeAsO}_{1-x} \mathrm{~F}_{x}$. Phys. Rev. Lett. 101, 057003 (2008).

14. Kuroki, K. et al. Unconventional pairing originating from the disconnected Fermi surfaces of superconducting $\mathrm{LaFeAsO}_{1-x} \mathrm{~F}_{x}$. Phys. Rev. Lett. 101, 087004 (2008).

15. Seo, K., Bernevig, A. B. \& Hu, J. Pairing symmetry in a two-orbital exchange coupling model of oxypnictides. Phys. Rev. Lett. 101, 206404 (2008).

16. Hu, J. P. \& Ding, H. Local antiferromagnetic exchange and collaborative Fermi surface as key ingredients of high temperature superconductors. Preprint at http://arxiv.org/abs/1107.1334 (2011).

17. Chen, C. T., Tsuei, C. C., Ketchen, M. B., Ren, Z. A. \& Zhao, Z. X. Integer and half-integer flux-quantum transitions in a niobium-iron pnictide loop. Nature Phys. 6, 260-264 (2010)

18. Hanaguri, T., Niitaka, S., Kuroki, K. \& Takagi, H. Unconventional s-wave superconductivity in $\mathrm{Fe}(\mathrm{Se}, \mathrm{Te})$. Science 328, 474-476 (2010).

19. Kuroki, K., Usui, H., Onari, S., Arita, R. \& Aoki, H. Pnictogen height as a possible switch between high- $T_{c}$ nodeless and low- $T_{c}$ nodal pairings in the iron-based superconductors. Phys. Rev. B 79, 224511 (2009).

20. Wang, F., Zhai, H. \& Lee, D-H. Nodes in the gap function of LaFePO, the gap function of the $\mathrm{Fe}(\mathrm{Se}, \mathrm{Te})$ systems, and the STM signature of the $\mathrm{s}_{ \pm}$pairing. Phys. Rev. B 81, 184512 (2010).

21. Thomale, R., Platt, C., Hanke, W. \& Bernevig, B. A. Why some iron-based superconductors are nodal while others are nodeless. Phys. Rev. Lett. 106, 187003 (2011).

22. Laad, M. S. \& Craco, L. Theory of multiband superconductivity in iron pnictides. Phys. Rev. Lett. 103, 017002 (2009).

23. Wang, F., Zhai, H. \& Lee, D-H. Nodes in the gap function of LaFePO, the gap function of the $\mathrm{Fe}(\mathrm{Se}, \mathrm{Te})$ systems, and the STM signature of the $s_{ \pm}$pairing. Phys. Rev. B 81, 184512 (2010).

24. Suzuki, K., Usui, H. \& Kuroki, K. Possible three dimensional nodes in the $s_{ \pm}$ superconducting gap of $\mathrm{BaFe}_{2}\left(\mathrm{As}_{1-x} \mathrm{P}_{x}\right)_{2}$. J. Phys. Soc. Jpn 80, 013710 (2011).
25. Su, Y., Setty, C., Wang, Z. \& Hu, J. Inter-layer superconducting pairing induced c-axis nodal lines in iron-based superconductors. Preprint at http://arxiv.org/abs/1110.0695 (2011).

26. Ye, Z. R. et al. Phosphor induced significant hole-doping in ferropnictide superconductor $\mathrm{BaFe}_{2}\left(\mathrm{As}_{1-x} \mathrm{P}_{x}\right)_{2}$. Preprint at http://arxiv.org/abs/ 1105.5242 (2011)

27. Yoshida, T. et al. Two-dimensional and three-dimensional Fermi surfaces of superconducting $\mathrm{BaFe}_{2}\left(\mathrm{As}_{1-x} \mathrm{P}_{x}\right)_{2}$ and their nesting properties revealed by angle-resolved photoemission spectroscopy. Phys. Rev. Lett. 106, 117001 (2011).

28. Shimojima, T. et al. Orbital-independent superconducting gaps in iron-pnictides. Science 332, 564-567 (2011).

29. Zhang, Y. et al. Out-of-plane momentum and symmetry-dependent energy gap of the pnictide $\mathrm{Ba}_{0.6} \mathrm{~K}_{0.4} \mathrm{Fe}_{2} \mathrm{As}_{2}$ superconductor revealed by angle-resolved photoemission spectroscopy. Phys. Rev. Lett. 105, 117003 (2010).

\section{Acknowledgements}

The authors thank J. P. Hu and X. H. Chen for discussions, and thank D. H. Lu for the experimental assistance at SSRL. This work is supported in part by the National Science Foundation of China, Ministry of Education of China, Shanghai Municipal Science and Technology Committee and the National Basic Research Program of China (973 Program) under grant Nos 2012CB921400, 2011CB921802 and 2011CBA00112. SSRL is operated by the US Department of Energy, Office of Basic Energy Science, Divisions of Chemical Sciences and Material Sciences.

\section{Author contributions}

Y.Z., Q.Q.G., Z.R.Y., F.C., M.X. and B.P.X. made ARPES measurements. Z.R.Y. and J.J. conducted sample characterization measurements. Z.R.Y. and Y.Z. grew the samples and analysed the ARPES data. D.L.F. and Y.Z. wrote the paper. D.L.F. is responsible for the infrastructure, project direction and planning.

\section{Additional information}

The authors declare no competing financial interests. Supplementary information accompanies this paper on www.nature.com/naturephysics. Reprints and permissions information is available online at www.nature.com/reprints. Correspondence and requests for materials should be addressed to D.L.F. 